\title{
ON THE NORMALITY OF SOME CAYLEY DIGRAPHS WITH VALENCY 2
}

\author{
Mehdi Alaeiyan $^{1}$ And Mohsen Ghasemi ${ }^{2}$ \\ ${ }^{1}$ Department of Mathematics, Iran University of Science and Technology, \\ Narmak, Tehran 16844, Iran \\ ${ }^{2}$ Department of Mathematics, Urmia university, \\ Urmia, 57135, Iran m.ghasemi@mail.urmia.ac.ir
}

\begin{abstract}
We call a Cayley digraph $\Gamma=\operatorname{Cay}(G, S)$ normal for $G$ if $R(G)$, the right regular representation of $G$, is a normal subgroup of the full automorphism group $\operatorname{Aut}(\Gamma)$ of $\Gamma$. In this paper we determine the normality of Cayley digraphs of valency 2 on the groups of order $p q$ and also on non-abelian finite groups $G$ such that every proper subgroup of $G$ is abelian.

Key words: Cayley digraph, normal Cayley digraph, automorphism group.
\end{abstract}

2000 Mathematics Subject Classification: 05C25, $20 \mathrm{~B} 25$.

Received: 23-06-2011, revised: 16-08-2011, accepted: 23-09-2011. 